\title{
Absence of commensurate ordering at the polarization flop transition in multiferroic $\mathrm{DyMnO}_{3}$
}

\author{
J. Strempfer, ${ }^{1}$ B. Bohnenbuck, ${ }^{2}$ M. Mostovoy, ${ }^{3}$ N. Aliouane, ${ }^{4}$ D. N. Argyriou, ${ }^{4}$ F. Schrettle, ${ }^{5}$ J. Hemberger ${ }^{5}$ \\ A. Krimmel, ${ }^{5}$ and M. v. Zimmermann ${ }^{1}$ \\ ${ }^{1}$ Hamburger Synchrotronstrahlungslabor HASYLAB at Deutsches Elektronen-Synchrotron DESY, 22605 Hamburg, Germany \\ ${ }^{2}$ Max-Planck-Institut für Festkörperforschung, 70569 Stuttgart, Germany \\ ${ }^{3}$ Zernike Institute for Advanced Materials, University of Groningen, 9747 AG Groningen, The Netherlands \\ ${ }^{4}$ Hahn-Meitner-Institut, 14109 Berlin, Germany \\ ${ }^{5}$ Experimentalphysik V, Universität Augsburg, 86159 Augsburg, Germany \\ (Received 21 February 2007; revised manuscript received 4 April 2007; published 5 June 2007)
}

\begin{abstract}
Ferroelectric spiral magnets $\mathrm{DyMnO}_{3}$ and $\mathrm{TbMnO}_{3}$ show similar behavior of electric polarization in applied magnetic fields. Studies of the field dependence of lattice modulations on the contrary show a completely different picture. Whereas in $\mathrm{TbMnO}_{3}$ the polarization flop from $P \| c$ to $P \| a$ is accompanied by a sudden change from incommensurate to commensurate wave vector modulation, in $\mathrm{DyMnO}_{3}$ the wave vector varies continuously through the flop transition. This smooth behavior may be related to the giant magnetocapacitive effect observed in $\mathrm{DyMnO}_{3}$.
\end{abstract}

DOI: 10.1103/PhysRevB.75.212402

The ability to control ferroelectric polarization $(\boldsymbol{P})$ with an applied magnetic field $(\boldsymbol{H})$ in manganite perovskites and related materials has caused a resurgence of interest in magnetoelectric phenomena. One of the most striking effects is the fivefold increase of the dielectric constant of $\mathrm{DyMnO}_{3}$ in magnetic field, named giant magnetocapacitance. ${ }^{1}$ The key to this unprecedented sensitivity is the spiral magnetic ordering stabilized by competing exchange interactions, which forces positive and negative ions to shift in opposite directions and which can be rather easily influenced by applied magnetic fields.

Unlike conventional ferroelectrics, in the $R \mathrm{MnO}_{3}$ manganite perovskites such as $R=\mathrm{Tb}$ and Dy the emergence of ferroelectricity ${ }^{2,3}$ arises from the peculiar coupling of the lattice to a spiral ordering of Mn spins. ${ }^{4-6}$ Spiral ordering is defined by the wave vector $\boldsymbol{\kappa}$ and the axis $\boldsymbol{e}$ around which the spins rotate. For $R=\mathrm{Tb}$ and Dy, these two vectors are perpendicular to each other. ${ }^{4}$ The coupling of a uniform electric polarization $\boldsymbol{P}$ to an inhomogeneous magnetization $\boldsymbol{M}$ is phenomenologically described by a term linear in the gradient $\boldsymbol{\nabla} \boldsymbol{M}$, the so-called Lifshitz invariant. Such a coupling breaks the inversion symmetry of the crystal lattice in the spiral magnetic state and induces the direction of the polarization $\boldsymbol{P}=\gamma \chi M_{1} M_{2}[\boldsymbol{e} \times \boldsymbol{\kappa}]$, where $\gamma$ and $\chi$ are a coupling constant and the dielectric susceptibility, and $M_{1}$ and $M_{2}$ are amplitudes of the magnetic moments in directions perpendicular to $\boldsymbol{e}{ }^{6}$ For $R=\mathrm{Tb}$ and Dy, $\boldsymbol{\kappa}$ is parallel to the $b$ axis and $\boldsymbol{e}$ is parallel to the $a$ axis so that the ferroelectric polarization induced below the spiral ordering temperature $T_{C}$ is parallel to the $c$ axis $(P \| c) .^{2}$

The application of magnetic field parallel either to the $a$ $(H \| a)$ or $b$ axis $(H \| b)$ leads to a flop of the polarization from $P \| c$ to $P \| a$. The flop in the polarization is interpreted as the flop of the vector $\boldsymbol{e}$ from the $a$ (spins within the $b c$ plane) to the $c$ direction (spins within the $a b$ plane). For $R=\mathrm{Tb}$, these field-induced flops of $\boldsymbol{P}$ occur at critical fields of $H_{C}^{a} \sim 8 \mathrm{~T}$ and $H_{C}^{b} \sim 4.5 \mathrm{~T}$ at $4 \mathrm{~K}$ for field parallel to the $a$ and $b$ axes, respectively, and are associated with a first-order transition from an incommensurate (IC) low-field magnetic ordering to a commensurate $(\mathrm{CM})$ high-field phase. The $H-T$ phase diagram of DyMnO${ }_{3}$ is very similar to that of $\mathrm{TbMnO}_{3}$, with the same characteristic flops of $\boldsymbol{P}$ but at lower critical fields, $H_{C}^{a} \sim 6.5 \mathrm{~T}$ and $H_{C}^{b} \sim 1 \mathrm{~T}$ at $2 \mathrm{~K}^{3}$

In this Brief Report, we show that although the phase diagrams of $R=\mathrm{Dy}$ and Tb may be qualitatively similar, their structural behavior at $H_{C}$ is completely different. We find that for $\mathrm{DyMnO}_{3}$, the polarization flop is not associated with a transition to a CM phase as in the case of $R=\mathrm{Tb}$, but rather the magnitude of the wave vector changes very little across $H_{C}$ for both $H \| a$ and $H \| b$ configurations. We argue that the magnitude of the incommensurability for $R=$ Dy does not lie sufficiently close to a $\mathrm{CM}$ value, as opposed to $R=\mathrm{Tb}$, making the IC high-field phase energetically more favorable.

For $R=\mathrm{Dy}, \mathrm{Mn}$ spins order to form a longitudinal spindensity wave below $T_{N} \sim 39 \mathrm{~K}$ with wave vector $\boldsymbol{\kappa}=\delta_{m} \mathbf{b}^{*}$, with $\delta_{m}^{\mathrm{Mn}} \sim 0.36, \ldots, 0.385$ (Ref. 3) determined on the basis of lattice reflections with $\delta_{l}=2 \delta_{m}$ (Refs. 3, 7, and 8) that arise from a coupling of the IC magnetic ordering to the lattice via a quadratic magnetoelastic coupling. ${ }^{9}$ With further cooling, $\delta_{m}^{\mathrm{Mn}}$ decreases down to $T_{C}=19 \mathrm{~K}$, where a second transition into the spiral phase occurs. Coincident with the transition to a spiral phase, a spontaneous electric polarization parallel to the $c$ axis is found. ${ }^{2}$ Below $T_{N}^{\mathrm{Dy}}=5 \mathrm{~K}$, Dy magnetic moments order commensurately with propagation vector $\frac{1}{2} \mathbf{b}^{*}{ }^{8}$ In the study presented here, structural first- and second-harmonic reflections related to the magnetic first-harmonic reflections are investigated.

Single-crystalline samples were prepared using the floating zone technique at the HMI. Details of sample preparation and characterization are given elsewhere. ${ }^{8}$ Due to the high neutron absorption cross section of Dy, in-field neutron diffraction is not ideal to investigate field-induced magnetostructural transitions in $\mathrm{DyMnO}_{3}$. Rather to investigate the structural response to the field-induced polarization flop, we have utilized in-field synchrotron x-ray diffraction with the two field configurations $H \| a$ and $H \| b$. Measurements with $H \| a$ were performed at beamline X21 at the National Synchrotron Light Source at Brookhaven National Laboratory 
with a photon energy of $9.5 \mathrm{keV}$, using a $13 \mathrm{~T}$ Oxford cryomagnet with vertical field. Measurements in the $H \| b$ configuration were carried out at beamline BW5 at HASYLAB with a photon energy of $100 \mathrm{keV}$ using a $10 \mathrm{~T}$ Cryogenics cryomagnet with horizontal field. Magnetization measurements were performed with a Physical Properties Measurement System (PPMS) on a $14.5 \mathrm{mg} \mathrm{DyMnO}_{3}$ sample. The spontaneous electric polarization as a function of field and temperature was determined from the pyrocurrent recorded in a PPMS using an electrometer.

Measurements of the spontaneous electric polarization performed on the same samples used in our diffraction experiments in magnetic-field configurations $H \| a$ and $H \| b$ [insets in Figs. 1(c) and 2(a)] confirm that spontaneous ferroelectric polarization is present below $T_{C}=19 \mathrm{~K}$, as already reported in Ref. 3. The application of $H \| b$ below $T_{C}$ (Dy) results in the suppression of $P \| c$ [inset of Fig. 1(c)]. Kimura et al. show, in addition, that this decrease is accompanied by an increase in $P \| a$, indicative of the flop in the ferroelectric polarization. ${ }^{3}$

In Figs. 1(a) and 1(b), we show the temperature dependence of the wave vector and integrated intensity of the second-harmonic reflection $(0,4-2 \delta, 0)$, both for decreasing and increasing temperature. The dependences are compared for zero field $(P \| c)$ and $\mu_{0} H \| b=10 \mathrm{~T}(P \| a)$. In both data sets, we find a significant hysteresis in the intensity, as well as in the magnitude of $\delta$ around $T_{C}=18 \mathrm{~K}$, which is associated with the onset of ferroelectricity. However, despite the fact that $\boldsymbol{P}$ lies along different axes for 0 and $10 \mathrm{~T}$, we observed no significant change between these two measurements. In Figs. 1(c) and 1(d), we show the field dependence of $\delta$ and of the integrated intensity of the same reflection at $T=2 \mathrm{~K}$. Here, we find a small initial increase of $\delta$ up to $\mu_{0} H_{C}^{b}=1 \mathrm{~T}$ where the flop in polarization is found, while the intensity of the same reflection shows a steady increase with increasing field. Above $H_{C}^{b}$, we find a small decrease of $\delta$ with increasing field. This behavior is in sharp contrast to $\mathrm{TbMnO}_{3}$, where $\delta$ varies slowly with increasing field and locks in above $H_{C}^{b}$ into a $\mathrm{CM}$ value of $\boldsymbol{\kappa}=\frac{1}{4} \mathbf{b}^{*}$ at $H_{C}^{b}{ }^{10}$

In Figs. 1(e) and 1(f), the temperature dependence of $\delta$ and the intensities of first- and second-harmonic reflections is shown for data measured in field cooling with $\mu_{0} H \| b$ $=2.5 \mathrm{~T}$. Here, we find a different behavior in the intensities of IC reflections. For wave vector along the $b^{*}$ direction $(0$, $4-2 \delta, 0$ ), a strong hysteresis is observed in its intensity as a function of temperature, whereas this hysteresis is absent for wave vectors that are mainly along the $c^{*}$ direction $[(0, \delta, 5)$ and $(0,2 \delta, 5)]$ [Fig. 1(f)]. Nevertheless, $\delta$ and its hysteresis are the same for all reflections [Fig. 1(e)]. The described intensity behavior is similar to what we have recently observed in zero-field temperature-dependent measurements using resonant $\mathrm{x}$-ray scattering from a single crystal of DyMnO${ }_{3} \cdot{ }^{11}$ In these measurements, a similar hysteresis was observed to be associated with the induced ordering of Dy spins with the same propagation vector as that for the Mn spin ordering. Finally, for this field configuration, we note that at $2.5 \mathrm{~T}$, the polarization flop from $P \| a$ to $P \| c$ is expected with increasing temperature at $T_{P F} \sim 12 \mathrm{~K}$ [inset in Fig. 1(c)]. However, our diffraction measurements find no anomaly either on the temperature dependence of the wave
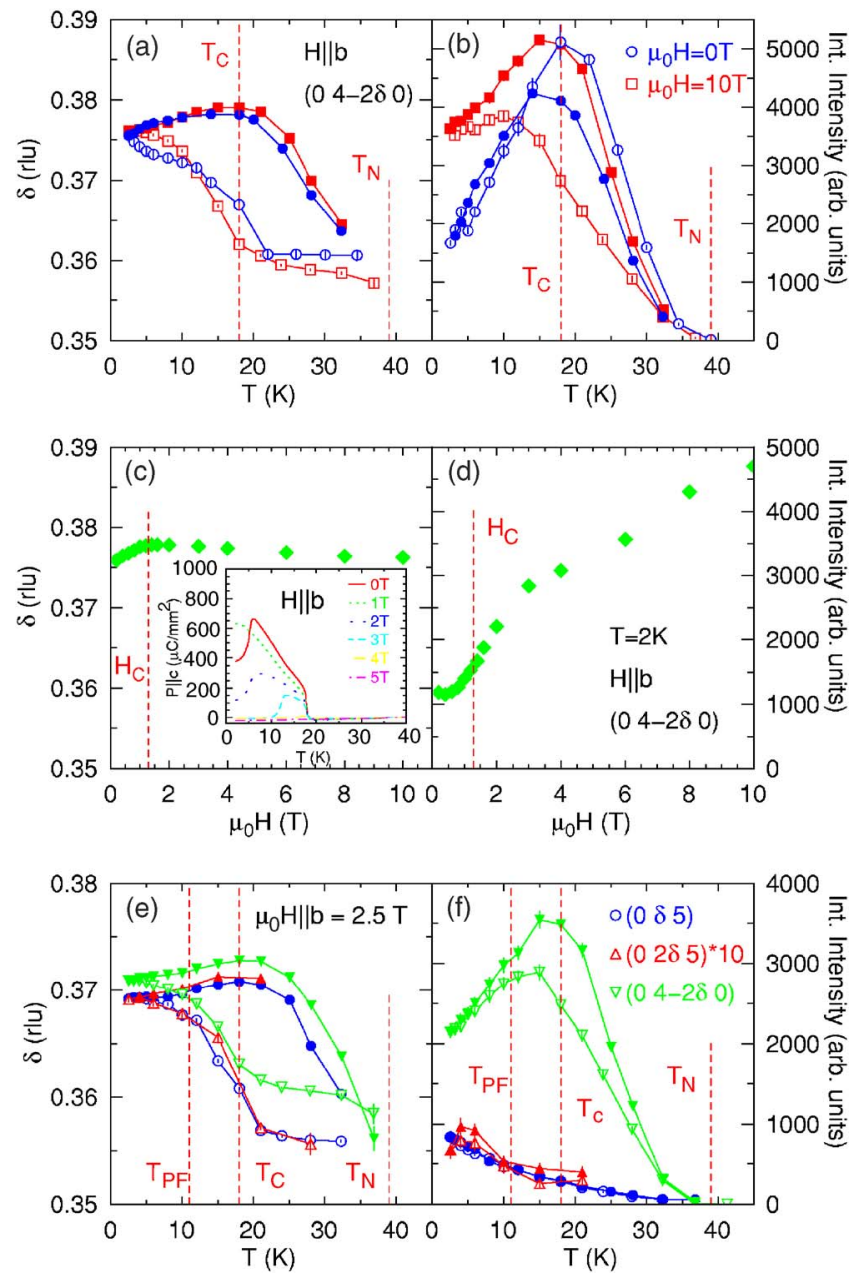

FIG. 1. (Color online) Temperature dependence of (a) the incommensurability $\delta$ and (b) the respective intensities of the $(0$, $4-2 \delta, 0)$ structural reflection. Data are shown for $\mu_{0} H=0 \mathrm{~T}$ and $\mu_{0} H \| b=10 \mathrm{~T}$ for decreasing (open symbols) and increasing (closed symbols) temperature. In (c) and (d), $\delta$ and intensity variations as function of magnetic field, respectively, are shown for a sample temperature of $T=2 \mathrm{~K}$, in the same axes range as in (a) and (b). In the inset, spontaneous electric polarization $P \| c$ is shown as function of temperature for magnetic-field orientation $H \| b$. Temperature dependence of (e) wave vector and (f) the respective intensities for $\mu_{0} H \| b=2.5 \mathrm{~T}$ and wave vectors $(0, \delta, 5),(0,2 \delta, 5)$, and $(0,4-2 \delta$, $0)$ for decreasing (open symbols) and increasing (closed symbols) temperature. The $(0,2 \delta, 5)$ intensities in (f) are multiplied by a factor of 10 .

vector or in the intensities at this temperature as was found in $\mathrm{TbMnO}_{3}$.

We now turn our attention to measurements conducted in the $H \| a$ configuration. In Figs. 2(a) and 2(b), the field dependence of the intensity and wave vector of the first- and second-harmonic reflections $(0,1-\delta, 5)$ and $(0,2 \delta, 5)$ is shown, measured at $T=8 \mathrm{~K}$ above the ordering temperature of Dy of $T_{N}^{\mathrm{Dy}} \sim 5 \mathrm{~K}$. The second-harmonic reflection decreases in intensity with increasing field and vanishes at $5 \mathrm{~T}$, while the first-harmonic reflection appears only at a field of $4 \mathrm{~T}$ and saturates in intensity at $7 \mathrm{~T}$. This behavior is not directly related to the polarization flop but reflects a variation 


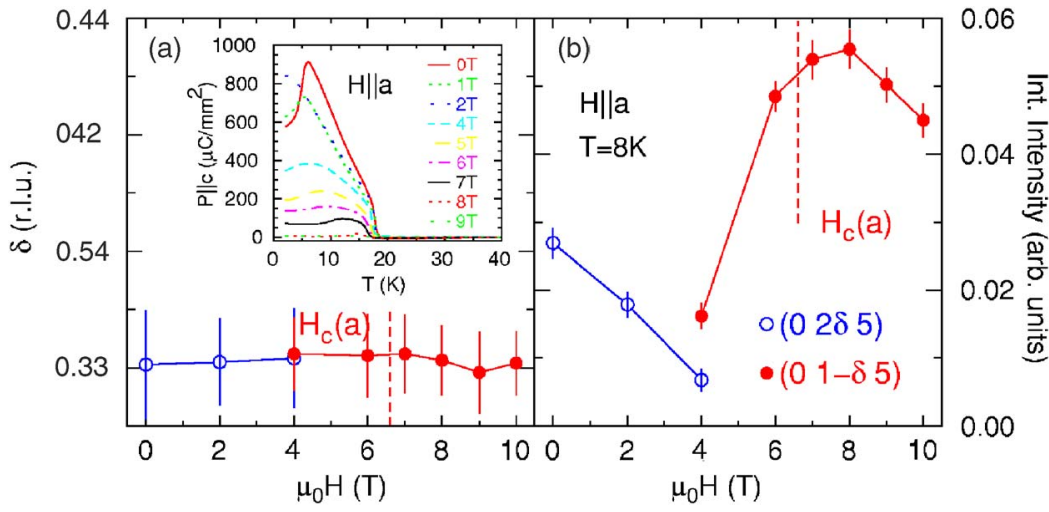

FIG. 2. (Color online) Field dependences with field $H \| a$ of (a) the incommensurability and (b) the integrated intensity measured at $T=8 \mathrm{~K}$. The error bars in (a) represent the half-width at half maximum of the superlattice reflection. In the inset, spontaneous electric polarization $P \| c$ is shown as function of temperature for magnetic field $H \| a$. of the modulation direction of the strain wave leading to the superlattice reflections. However, the most remarkable behavior is found for the field dependence of the wave vector. Here, the wave vector of the first-harmonic reflection does not change significantly through the polarization flop transition at $\mu_{0} H_{C}^{a} \sim 6.5 \mathrm{~T} .{ }^{3}$ Again this is in sharp contrast to the behavior of $\mathrm{TbMnO}_{3}$, where a discontinuous transition to a $\mathrm{CM}$ phase is found.

Below $6 \mathrm{~K}$, Dy spins order commensurately with $\delta_{m}^{\mathrm{Dy}}=\frac{1}{2}$. Previously we have argued that the lattice distortion associated with this magnetic ordering is not $\mathrm{CM}$ but rather IC with $\delta_{l} \sim 0.1 \mathbf{b}^{*}{ }^{8}$ In our measurements we found the half-integer magnetic reflection to be extremely weak and we focused our attention to the lattice $\delta_{l} \sim 0.1$ satellite measured at $(0,0.9$, 5). In Fig. 3(a), we show a series of scans at different fields applied along the $a$ axis at $3 \mathrm{~K}$, which show the rapid suppression of the $\delta_{l} \sim 0.1$ satellite which vanishes for fields $\mu_{0} H \| a>1 \mathrm{~T}$. The CM magnetic reflection $(0,0.5,5)$ is only measurable at zero field and can no longer be observed at $1 \mathrm{~T}$. The temperature dependence of the intensity and wavevector of the $(0,0.9,5)$ reflection is shown in Figs. 3(b) and 3(c), revealing the rapid suppression of the intensity of this reflection at low temperature in accordance with the appearance of ferromagnetic order [inset Fig. 3(c)], indicating an easy axis along the $b$ direction. The suppression of this reflection with magnetic field is analogous to a similar behavior found for $R=\mathrm{Tb}$ which coincided with the ferromagnetic ordering of $\mathrm{Tb}$ spins for the same field size and configuration. ${ }^{10}$

The polarization flop is driven by the field-induced flop of the axis of rotation of the magnetic spiral $\boldsymbol{e}$. Its direction is determined by magnetic anisotropy terms, which to lowest order of the free-energy expansion in powers of magnetization of Mn spins have the form $\sum_{\alpha=a, b, c} a_{\alpha} M_{\alpha}^{2}$. Phenomenologically, the spin-flop transition results from the field dependence of the coefficients $a_{\alpha}$. Below the critical field $H_{C}, a_{b}$ $<a_{c}<a_{a}$, which favors spins rotating in the $b c$ plane $(e \| a)$, while above $H_{C}, a_{b}<a_{a}<a_{c}$, favoring the rotation in the $a b$ plane $(e \| c)$.

From the perspective of symmetry, there is no restriction that the high-field spiral phase must be CM. In this view, the fact that there is a magnetoelastic phase transition to a $\mathrm{CM}$ phase associated with the flops in the polarization for $R$ $=$ Tb would appear to be a special case, especially when compared to $R=$ Dy where we find no such transition at $H_{C}$. The difference in behavior between these two multiferroics is not of fundamental nature but rather simply lies in the magnitude of the incommensurability. For $R=\mathrm{Tb}, \delta_{m}=0.28 \mathrm{rlu}$ is close to the $\mathrm{CM}$ value of $\frac{1}{4}$. For this $\mathrm{CM}$ value of the wave vector, the amplitude of the Mn magnetic moment is not modulated $^{10}$ and may thus be energetically more favorable than an IC amplitude modulated phase. In the phenomenological approach, the CM state with $\delta=0.25$ rlu is stabilized by $M^{4}$ terms in the Landau expansion. For $R=\mathrm{Dy}$, the value of $\delta=0.38 \mathrm{rlu}$ is further away from a CM value $\left(\frac{1}{3}\right)$ and thus a transition to it is not favorable energetically. Therefore, the Mn spin spiral may indeed flop as expected but without a
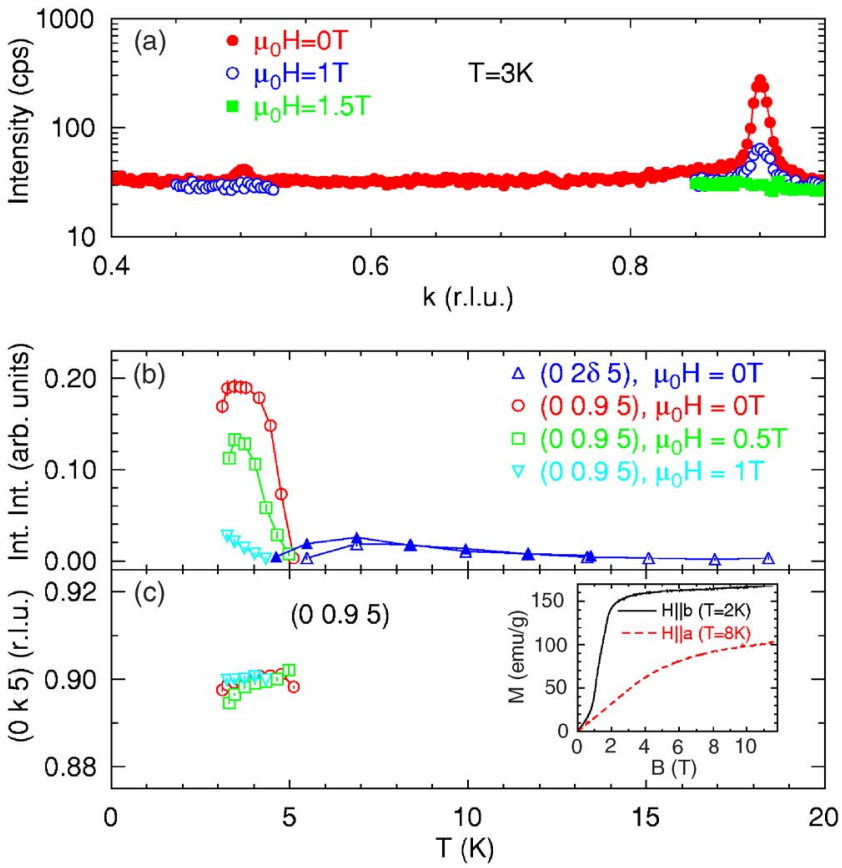

FIG. 3. (Color online) (a) Scans along $(0, k, 5)$ at $T=3 \mathrm{~K}$ for different applied magnetic fields with intensity in logarithmic scale. (b) Temperature dependence as function of field $H \| a$ of the integrated intensity of the superlattice reflections $(0,0.9,5)$ in the Dy ordered phase and the second-harmonic reflection $(0,2 \delta, 5)$ due to $\mathrm{Mn}$ order. Open and closed symbols represent increasing and decreasing temperature, respectively. In (c) the wave vector of the $(0$, $0.9,5)$ reflection is shown as function of temperature for different fields. The inset shows magnetization data of $\mathrm{DyMnO}_{3}$ for $H \| a$ and $H \| b$. 
change in $\delta .{ }^{13}$ Clearly, here the modulation period in real space is much shorter, and the amplitude of the Mn magnetic moment is modulated.

A significant difference between the two multiferroics is found in the behavior of the complex magnetic ordering of $R$ ions. For $R=\mathrm{Tb}, \mathrm{Tb}$ spins are induced to order along the $a$ axis below $T_{N}$ with the same periodicity as Mn spins, and below $T_{N}^{\mathrm{Tb}}=7 \mathrm{~K}$, they order separately with $\delta_{m}^{\mathrm{Tb}}=0.42 \mathrm{rlu}$. At low temperatures, when $H \| a \sim 1 \mathrm{~T}$, Tb spins are aligned ferromagnetically, while for field along the $b$ axis, $\delta_{m}^{\mathrm{Tb}}$ jumps discontinuously to a $\mathrm{CM}$ value of $\frac{1}{3}$ at $1.75 \mathrm{~T} .{ }^{10}$ The behavior for $R=\mathrm{Dy}$ is much simpler, as below $T_{N}^{\mathrm{Dy}}=6 \mathrm{~K}$ the Dy spins order with $\delta_{m}^{\mathrm{Dy}}=\frac{1}{2}$. Here, the suppression of the IC reflections together with the magnetization data indicates a melting of the antiferromagnetic Dy ordering, a behavior different from that of $\mathrm{TbMnO}_{3}$.

On the basis of the CM ordering of Mn spins for the $P \| a$ phase for $R=\mathrm{Tb}$, it has been suggested that ferroelectricity may arise in the absence of a spiral magnetic ordering. Here, an exchange striction mechanism proposed from competing ferromagnetic and antiferromagnetic superexchange interactions predicts a $P \| a$ phase for a CM ordering with $\delta=\frac{1}{4}$. This model holds strictly for a CM ordering and suggests that for $R=$ Dy the spiral phase must be maintained at high fields to support a ferroelectric state.

The smooth magnetic-field dependence of the spiral wave vector in $\mathrm{DyMnO}_{3}$ at the spin-flop transition may explain the large increase of the dielectric constant $\varepsilon_{a}$ observed in this material. ${ }^{1,3}$ If higher-order terms in the Landau expansion of free energy could be neglected, then at the spin-flop transition the magnetic excitation spectrum of the spiral would acquire a zero mode, since for $a_{a}\left(H_{C}\right)=a_{c}\left(H_{C}\right)$ there is a freedom to rotate the spiral plane around the $b$ axis. This mode can be excited by electric field $E \| a$ normal to the spiral $b c$ plane and is the electromagnon studied in Ref. 12. Its softening at $H=H_{C}$ would result in a divergence of the static dielectric susceptibility $\varepsilon_{a}$. In reality, due to higherorder terms in the Landau expansion, the spin-flop transition is of first order, the softening of the magnetic mode is not complete, and the peak value of the dielectric constant is finite. Still, in $\mathrm{DyMnO}_{3}$ this transition is close to a secondorder one in the sense that the spirals above and below the critical field are essentially the same except for their orientation. The softness of the spiral magnetic ordering at the critical field may be the reason behind the large magnetocapacitance observed in $\mathrm{DyMnO}_{3}$, which becomes truly gigantic close to the tricritical point at the crossing of the collinear and two spiral phases with $P \| c$ and $P \| a$, where higher-order terms are small. In $\mathrm{TbMnO}_{3}$, the spin-flop transition is strongly discontinuous due to the concomitant IC-CM transition, which limits the growth of the dielectric constant.

In summary, in-field synchrotron x-ray diffraction measurements from a $\mathrm{DyMnO}_{3}$ single crystal have shown that there is no change of the wave vector $\delta$ associated with the flop of the ferroelectric polarization $\boldsymbol{P}$ at $H_{C}$ for both $H \| a$ and $H \| c$. This is in sharp contrast to similar measurements reported for $\mathrm{TbMnO}_{3}$, where a transition to a CM phase is found at $H_{C}$ for the same field configurations. We argue that the magnitude of the incommensurability for $R=$ Dy does not lie sufficiently close to a CM value, as opposed to $R=\mathrm{Tb}$, making the IC high-field phase energetically more favorable.

We would like to thank C. S. Nelson for the assistance with the experiment at NSLS. Work at Brookhaven was supported by the U.S. Department of Energy, Division of Materials Science, under Contract No. DE-AC02-98CH10886.
${ }^{1}$ T. Goto, T. Kimura, G. Lawes, A. P. Ramirez, and Y. Tokura, Phys. Rev. Lett. 92, 257201 (2004).

${ }^{2}$ T. Kimura, T. Goto, H. Shintani, K. Ishizaka, T. Arima, and Y. Tokura, Nature (London) 426, 55 (2003).

${ }^{3}$ T. Kimura, G. Lawes, T. Goto, Y. Tokura, and A. P. Ramirez, Phys. Rev. B 71, 224425 (2005).

${ }^{4}$ M. Kenzelmann, A. B. Harris, S. Jonas, C. Broholm, J. Schefer, S. B. Kim, C. L. Zhang, S.-W. Cheong, O. P. Vajk, and J. W. Lynn, Phys. Rev. Lett. 95, 087206 (2005).

${ }^{5}$ H. Katsura, N. Nagaosa, and A. V. Balatsky, Phys. Rev. Lett. 95, 057205 (2005).

${ }^{6}$ M. Mostovoy, Phys. Rev. Lett. 96, 067601 (2006).

${ }^{7}$ T. Kimura, S. Ishihara, H. Shintani, T. Arima, K. T. Takahashi, K. Ishizaka, and Y. Tokura, Phys. Rev. B 68, 060403(R) (2003).
${ }^{8}$ R. Feyerherm, E. Dudzik, N. Aliouane, and D. N. Argyriou, Phys. Rev. B 73, 180401(R) (2006).

${ }^{9}$ M. B. Walker, Phys. Rev. B 22, 1338 (1980).

${ }^{10}$ N. Aliouane, D. N. Argyriou, J. Strempfer, I. Zegkinoglou, S. Landsgesell, and M. v. Zimmermann, Phys. Rev. B 73, 020102(R) (2006).

${ }^{11}$ O. Prokhnenko, R. Feyerherm, E. Dudzik, S. Landsgesell, N. Aliouane, L. C. Chapon, and D. N. Argyriou, Phys. Rev. Lett. 98, 057206 (2007).

${ }^{12}$ A. Pimenov, A. A. Mukhin, V. Yu Ivanov, V. D. Travkin, A. M. Balbashov, and A. Loidl, Nat. Phys. 2, 97 (2006).

${ }^{13}$ We note that a value of $\delta=\frac{1}{3}$ is observed for $\mathrm{Tb}_{1-x} \mathrm{Dy}_{x} \mathrm{MnO}_{3}$, see Arima et al., Phys. Rev. Lett. 96, 097202 (2006). 